\title{
DEMANDA DE INTERVENÇÕES DE ENFERMAGEM A PACIENTES SOB CUIDADOS INTENSIVOS: NAS - NURSING ACTIVITIES SCORE
}

\author{
Demand for nursing interventions to patients under intensive care: nas - nursing activities \\ score \\ Demanda de intervenciones de enfermería a pacientes que necesitsan cuidados intensivos: \\ nas - nursing activities score
}

Manuella Carvalho Feitosa ${ }^{1}$

Illoma Rossany Lima Leite²

Grazielle Roberta Freitas da Silva ${ }^{3}$

\section{RESUMO}

Na assistência de enfermagem a pacientes críticos, a utilização de instrumentos capazes de quantificar a demanda de cuidados é fundamental para adequação da equipe de enfermagem e, com isso, melhorar o planejamento e a qualidade da assistência. Estudo descritivo e quantitativo, realizado de setembro a outubro/2011, com objetivo de avaliar a demanda de cuidados de enfermagem pela aplicação do Nursing Activities Score (NAS). Amostra de 45 pacientes, maioria do sexo feminino (64,4\%), média de 51,4 anos recrutados em duas Unidades Intensivas de um Hospital Público em Teresina-Piauí. Foram realizadas 328 medidas do NAS, com média do escore total de $67,3 \%$, variando de $39,2 \%$ a $133,7 \%$. Os itens mais pontuados foram referentes às categorias "Atividades básicas", "Tarefas administrativas e gerenciais" e "Suporte respiratório". Uma média do NAS elevada permitiu concluir que essa clientela apresentou acentuada necessidade de cuidados, indicando o NAS como um bom instrumento para gerenciar recursos humanos de enfermagem.

Palavras-chave: Enfermagem. Unidade de Terapia Intensiva. Cuidados de enfermagem.

\begin{abstract}
In the nursing care of critically ill patients the use of instruments capable of quantifying the demand for care is fundamental for the adequacy of nursing staff and thereby improves the quality of care and planning. Thus, the aim was to evaluate the demand for nursing care by the application of the Nursing Activities Score (NAS) in two intensive care units of a public hospital in Teresina, Piauí. This was a descriptive retrospective and quantitative study, conducted from September to October/2011. There was a sample of 45 patients, mostly female (64.4\%), with an average age of 51.4 years old. There were 328 measurements carried out from NAS, we obtained an average total score of $67.3 \%$, ranging from $39.2 \%$ to $133.7 \%$, and items related to the categories "Basic Activities", "Administrative and managerial tasks" and "Respiratory support" as highest scores. It is concluded that these clients had severe need for care, reflected by the high NAS average, representing a good tool to manage human resources in nursing.
\end{abstract}

Keywods: Nursing. Intensive Care Unit. Nursing Care.
En los cuidados de enfermería de los pacientes críticos, el uso de instrumentos capaces de cuantificar la demanda de cuidados es fundamental para la adecuación del equipo de enfermería y, así, mejorar la planificación y calidad de la atención en salud. El objetivo de la investigación era evaluar la demanda de cuidados de enfermería mediante la aplicación del Nursing Activities Score (NAS) en dos unidades de cuidados intensivos de un hospital público de Teresina, Piauí. Este estudio cuantitativo, descriptivo y retrospectivo fue realizado de Septiembre a Octubre/2011. Contó con una muestra de 45 pacientes, en su mayoría mujeres $(64,4 \%)$, con un promedio de 51,4 años de edad. Las 328 mediciones del NAS obtuvieron una puntuación media total de $67,3 \%$, que van desde el 39,2\% a 133,7\%, con artículos relacionados a las categorías: "Actividades básicas", "Tareas administrativas y de gestión" y "Soporte respiratorio", como puntuaciones más altas. Se concluye que estos clientes tenían necesidad grave para la atención, que se refleja por el alto promedio del NAS, lo que representa una buena herramienta para gestionar los recursos humanos en enfermería.

Palabras clave: Enfermería. Unidad de Cuidados Intensivos. Cuidados de Enfermería.

\footnotetext{
${ }^{1}$ Enfermeira. Pós-graduanda (Mestrado), Programa de Pós-Graduação Mestrado em Enfermagem da Universidade Federal do Piauí (UFPI) - Departamento de Enfermagem. Teresina -PI. Brasil. E-mail: manuellacf@hotmail.com; ${ }^{2}$ Enfermeira. Pós-graduanda (Mestrado), Programa de Pós-Graduação Mestrado em Enfermagem da Universidade Federal do Piauí (UFPI) - Departamento de Enfermagem. Teresina - PI. Brasil. E-mail: illomaa@hotmail.com; ${ }^{3}$ Enfermeira. Doutora em Enfermagem. Docente do Programa de Pós-Graduação Mestrado em Enfermagem da Universidade Federal do Piauí (UFPI) - Departamento de Enfermagem. Teresina - PI. Brasil. E-mail: grazielle_roberta@yahoo.com.br
} 


\section{INTRODUÇÃO}

Com o advento das Unidades de Terapia Intensiva (UTIs), houve alterações no tempo de permanência, nível de atenção e demanda de cuidados à medida que, com um maior número de recursos terapêuticos e tecnológicos à disposição, os pacientes tornaram-se mais graves e complexos para serem cuidados, necessitando de melhor quantificação e qualificação dos profissionais que atuam na área'.

Pacientes que necessitam de cuidados intensivos, além de requererem maior dispêndio físico (no que concerne à força de trabalho) por parte dos profissionais de enfermagem, em virtude da dependência e gravidade clínica, com a humanização da assistência nas UTIs, têm demandado também atenção dos enfermeiros para suprir suas necessidades de apoio emocional, relativas à terapêutica e prognósticos determinados, bem como as de seus familiares.

Contudo, proporcionar excelência no atendimento em UTI depende da qualidade dos cuidados de enfermagem prestados, quais sejam: suporte psicológico e emocional aos pacientes e familiares; monitorização à beira do leito por meio de eletrocardiograma, pressões hemodinâmica e arterial, oxigenação, parâmetros fisiológicos e monitorização da pressão intracraniana; cuidados na administração de drogas vasoativas; assistência no suporte mecânico da circulação e ventilação; controle hidroeletrolítico e acidobásico; suporte nutricional; avaliação neurológica; assistência ao paciente em parada cardiorrespiratória, entre outros ${ }^{2}$.

Assim, estudos têm verificado que a incorporação das novas tecnologias em saúde, especialmente na área da enfermagem intensiva, implicou um redimensionamento do espaço do cuidado e vem imprimindo mudanças gradativas nos cuidados prestados pelo enfermeiro e equipe, requerendo desse profissional em particular, como cuidador, habilidades da observação, comunicação, reflexão, aplicação dos conhecimentos científicos, além de se fazerem apreciações e tomada de decisões ${ }^{3,4}$.

Nas unidades de cuidados intensivos, por abrigarem pacientes críticos ou de alto risco, que necessitam de cuidados diretos e contínuos, pois seu quadro de saúde pode facilmente evoluir para a morte, édeterminante a presença de pessoal treinado e em número suficiente para que se estabeleçam adequados tratamento e cuidado $0^{3,5,6}$

Por conseguinte, pesquisas têm defendido o uso de sistemas de classificação de paciente versus trabalho de enfermagem, para que haja maior congruência entre as necessidades de cuidados dos pacientes e as horas de enfermagem disponíveis, sendo úteis para justificar a necessidade de um maior número de pessoal permanentemente na equipe ${ }^{4,7,8}$. Conquanto, é necessário ${ }^{1,6}$ que sejam utilizados instrumentos de medida de fácil aplicação, abrangentes, precisos e validados para a cultura brasileira, a fim de evitar vieses na aferição do fenômeno ou tornar o procedimento de aplicação demasiado cansativo.
0 emprego de instrumento capaz de avaliar a demanda de cuidados de enfermagem pelos pacientes possibilita uma otimização da relação custo-benefício na assistência à saúde, no contexto atual, por meio de um melhor dimensionamento do pessoal de enfermagem para suprir essa demanda. Destarte, sua aplicação imediatamente após a avaliação do paciente pode proporcionar um melhor planejamento da assistência, com base, também, na frequência e duração das intervenções de enfermagem em unidades de cuidados intensivos ${ }^{7,8}$.

Diante do exposto, a equipe de pesquisadoras optou por utilizar o Nursing Activities Score (NAS), originalmente escrito na língua inglesa, que foi traduzido, adaptado e validado para a língua portuguesa em 20027. Tratando-se de uma versão atualizada do Therapeutic Intervention Scoring System (TISS28), o NAS contempla atividades de caráter assistencial, de suporte à família e administrativas ${ }^{7}$. Esse instrumento foi escolhido devido a fácil aplicação e aos bons resultados, até 0 ano corrente, referente às medidas psicrométricas apresentados em outras pesquisas ${ }^{1,6-8}$, justificando a importância de efetuar estudos dessa natureza com foco na aplicação de instrumentos traduzidos e validados no Brasil.

0 NAS é composto de 23 itens, que agregam ao escore pontuações que variam de 1,2 a 32,0. É subdividido em sete grandes categorias: atividades básicas, suporte ventilatório, suporte cardiovascular, suporte renal, suporte neurológico, suporte metabólico e intervenções especíicas. Estas descrevem um conjunto de atividades de cuidados intensivos de enfermagem, cujo escore total varia de 0 a $176,8 \%$, e abrangem $80,8 \%$ do tempo gasto pelo profissional de enfermagem no cuidado do paciente durante as 24 horas $^{1,8}$.

A equipe de pesquisa considerou relevante avaliar a demanda de cuidados de enfermagem, especialmente em UTI, por sua grande possibilidade de se transformar em um ambiente tenso se houver sobrecarga ou superestimação da mão-de-obra dos profissionais, comprometendo a qualidade da assistência. Outrossim, determinar intervenções mais frequentes favorece o planejamento da assistência em UTI, podendo reproduzir realidades vivenciadas em unidades semelhantes.

Dentro dessa perspectiva, o objetivo geral do estudo foi avaliar a demanda de cuidados de enfermagem em UTIs, e os objetivos espećficos foram: caracterizar os pacientes internados nessas UTIs; aplicar o NAS, visando mensurar, em porcentagem de tempo, os cuidados requeridos pelos pacientes nessas unidades; e verificar a frequência dos cuidados de enfermagem segundo os indicadores do NAS.

\section{MÉTODO}

Estudo de abordagem quantitativa, desenvolvido em duas UTIs gerais adulto de um hospital público, com 8 leitos de internação cada uma, totalizando 16 leitos, na cidade de Teresina-PI.

As unidades amostrais incluíram os prontuários de pacientes internados, com três critérios de inclusão: internação do paciente no período da coleta; pacientes com idade igual ou superior a 18 
anos; e período mínimo de permanência do paciente na UTI de 48 horas, satisfatório para adequação e direcionamento do cuidado mais efetivo e organizado pela equipe de enfermagem. Readmissões foram excluídas do estudo.

Aplicando-se o cálculo amostral de população finita, com erro amostral de $5 \%$ e nível de confiança de $95 \%$, obtevese uma amostra de 45 prontuários na população investigada.

A coleta de dados foi executada pelo primeiro pesquisador, diariamente, no turno da tarde, por meio da consulta aos prontuários, para informações dos cuidados de enfermagem e intervenções terapêuticas a que os pacientes foram submetidos. Os dados foram coletados e organizados com a utilização de impresso dividido em duas partes: a primeira com dados demográficos e clínicos dos pacientes e a segunda configurada pelo NAS. Para fins de padronização, foram consideradas as informações referentes às 24 horas do dia anterior da coleta de dados, sendo possível coletar informações mais completas e fidedignas ${ }^{6-8}$.

Os dados coletados foram armazenados em um banco eletrônico criado em planilhas no programa Excel $₫$ versão 2000

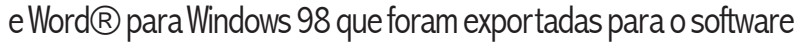
Statistical Package for the Social Sciences-SPSS $®$, versão 18.0, para posterior tabulação/tratamento estatístico. Os resultados foram organizados em tabelas simples com distribuição simples e percentual, discutidos à luz da literatura sobre o tema.

0 projeto foi submetido à apreciação do Comitê de Ética em Pesquisa da UFPI e aprovado com Certificado de Apresentação para Apreciação Ética (CAAE) nº 0115.0.045.000-11 (ANEXO A), seguindo aos parâmetros estabelecidos na resolução do Conselho Nacional de Saúde 196/1996. Quando o paciente não estava consciente, a autorização foi concedida pelo familiar ou responsável legal presente.

\section{RESULTADOS}

A amostra foi constituída por 45 pacientes. Conforme a Tabela 1, 29 eram do sexo feminino $(64,4 \%)$ e 16 do sexo masculino (35,5\%), com idade entre 18 e 96 anos e média de 51,4 anos. As faixas etárias que predominaram foram de 58 a 77 anos (37,8\%), seguidas de 18 a 37 anos (35,5\%).

Tabela 1 - Caracterização dos pacientes internados quanto ao sexo e faixa etária. Teresina - PI, $2011(n=45)$.

\begin{tabular}{llll}
\hline & & n & $\%$ \\
\hline Sexo & Fem. & 29 & 64,4 \\
& Masc. & 16 & 35,5 \\
Faixa etária & 18 a 37 & 16 & 35,5 \\
(anos) & 38 a 57 & 08 & 17,8 \\
& 58 a 77 & 17 & 37,8 \\
& 78 a 96 & 04 & 08,9 \\
\hline
\end{tabular}

Com relação aos aspectos clínicos, na Tabela 2 é observado que o tempo de internação variou de dois a 21 dias, com média de sete dias, sendo que $66,7 \%$ dos pacientes permaneceram internados de dois a oito dias. Quanto à procedência, a maioria, 20 pacientes ( $44,5 \%$ ), advieram das Unidades de Internação do próprio hospital e 15 pacientes (33,3\%), do Centro Cirúrgico. 0 tipo de internação, conforme dados dos prontuários, foi predominantemente clínica (62,3\%), tendo como principais motivos doenças cardiovasculares $(28,9 \%)$ e respiratórias (20,0\%). Já para as internações do tipo cirúrgica, somando-se as eletivas $(24,4 \%)$ com as de urgência (13,3\%), obteve-se um total de $37,7 \%$, sendo $35,5 \%$ motivadas por pós-operatório imediato, que foi o principal motivo de internação. Quanto ao desfecho clínico, 36 pacientes $(80,0 \%)$ sobreviveram e a taxa de mortalidade foi de $20,0 \%$, com nove casos evoluindo a óbito.

Tabela 2 - Caracterização dos pacientes internados nas UTIs quanto aos aspectos clínicos. Teresina - PI, 2011(n=45).

\begin{tabular}{|c|c|c|c|}
\hline & & $n$ & $\%$ \\
\hline \multicolumn{4}{|l|}{ Tempo de internação } \\
\hline & 2 a 8 dias & 30 & 66,7 \\
\hline & 9 a 15 dias & 12 & 26,7 \\
\hline & 16 a 21 dias & 03 & 6,6 \\
\hline \multicolumn{4}{|l|}{ Procedência* } \\
\hline & UI & 20 & 44,5 \\
\hline & $\mathrm{CC}$ & 15 & 33,3 \\
\hline & Outros & 10 & 22,2 \\
\hline \multicolumn{4}{|l|}{ Tipo de internação } \\
\hline & Clínica & 28 & 62,3 \\
\hline & Cirúrgica eletiva & 11 & 24,4 \\
\hline & Cirúrgica urgência & 06 & 13,3 \\
\hline \multicolumn{4}{|l|}{ Motivo da internação } \\
\hline & Doenças cardiovasculares & 13 & 28,9 \\
\hline & Pós-operatório imediato & 16 & 35,5 \\
\hline & Doenças respiratórias & 09 & 20,0 \\
\hline & Politrauma & 03 & 6,7 \\
\hline & Outros & 04 & 8,9 \\
\hline \multicolumn{4}{|l|}{ Desfecho clínico } \\
\hline & Sobrevivente & 36 & 80,0 \\
\hline & Não sobrevivente & 09 & 20,0 \\
\hline
\end{tabular}


Foram realizadas 328 medidas do NAS, e a carga de trabalho de enfermagem, apresentada em forma de percentual, como sugere o NAS, variou de $39,2 \%$ a $133,7 \%$, sendo a média total do NAS 67,3\% (DP=17,2).

Conforme a Tabela 3, os itens 1, 2, 3, 4, 6, 7 e 8 foram cuidados requeridos diariamente a todos os pacientes (100\%).
Os itens $5,9,10,11,12,17,21$ também foram pontuados com frequência elevada de 84,8\%, 77,4\%, 58,2\%, 77,1\%, $36,0 \%, 97,6 \%$ e $70,1 \%$, respectivamente. As menores frequências foram encontradas nos itens $13,15,16,18,19$, 20, 22 e 23. Já os indicadores do item 14 e do subitem 4c são cuidados não requeridos no período de coleta de dados.

Tabela 3 - Frequências de aplicação de medidas do NAS segundo seus indicadores. Teresina - PI, 2011 ( $n=$ 328).

\begin{tabular}{|c|c|c|}
\hline Indicadores do NAS & No. de Medidas do NAS & $\%$ \\
\hline 1. Monitorização e controles & 328 & 100,0 \\
\hline a. subitem 1 a & 135 & 41,2 \\
\hline b. subitem $1 \mathrm{~b}$ & 183 & 55,8 \\
\hline c. subitem $1 c$ & 10 & 3,0 \\
\hline 2. Investigações laboratoriais & 328 & 100,0 \\
\hline 3. Medicação, exceto drogas vasoativas & 328 & 100,0 \\
\hline 4. Procedimentos de higiene & 328 & 100,0 \\
\hline a. subitem $4 a$ & 204 & 62,2 \\
\hline b. subitem $4 b$ & 124 & 37,8 \\
\hline c. subitem $4 c$ & 0 & 0,0 \\
\hline 5. Cuidados com todos os drenos, exceto sonda gástrica & 278 & 84,8 \\
\hline 6. Mobilização e posicionamento & 328 & 100,0 \\
\hline a. subitem $6 a$ & 144 & 43,9 \\
\hline b. subitem $6 b$ & 183 & 55,8 \\
\hline c. subitem $6 c$ & 1 & 0,3 \\
\hline 7. Suporte e cuidados aos familiares e parentes & 328 & 100,0 \\
\hline a. subitem $7 \mathrm{a}$ & 318 & 97,0 \\
\hline b. subitem $7 b$ & 10 & 3,0 \\
\hline 8. Tarefas administrativas e gerenciais & 328 & 100,0 \\
\hline a. subitem $8 a$ & 256 & 78,0 \\
\hline b. subitem $8 b$ & 62 & 19,0 \\
\hline c. subitem $8 \mathrm{c}$ & 10 & 3,0 \\
\hline 9. Suporte respiratório & 254 & 77,4 \\
\hline 10. Cuidados comvias aéreas artificiais & 191 & 58,2 \\
\hline 11. Tratamento para melhora da função pulmonar & 253 & 77,1 \\
\hline 12. Medicação vasoativa, independente do tipo e da dose & 118 & 36,0 \\
\hline 13. Reposição intravenosa de grandes perdas de fluidos & 17 & 5,2 \\
\hline 14. Monitorização do átrio esquerdo & 0 & 0,0 \\
\hline 15. Reanimação cardiorrespiratória & 3 & 0,9 \\
\hline 16. Técnica de hemofiltração, técnicas dial'ticas & 7 & 2,1 \\
\hline 17. Medida quantitativa do débito urinário & 320 & 97,6 \\
\hline 18. Medida da pressão intracraniana & 2 & 0,6 \\
\hline 19. Tratamento da acidose/alcalose metabólica & 27 & 8,2 \\
\hline 20. Nutrição parenteral total & 5 & 1,5 \\
\hline 21. Alimentação enteral por sonda ou outra via gastrointestinal & 230 & 70,1 \\
\hline 22. Intervenção(ões) especifica(s) na UTI & 25 & 7,6 \\
\hline 23. Intervenções especificas fora da UTI & 20 & 6,1 \\
\hline
\end{tabular}

\section{DISCUSSÃO}

Os resultados desse estudo mostraram uma predominância de pacientes do sexo feminino $(64,4 \%)$, contrariando a maioria dos estudos realizados em UTI, nos quais há um predomínio de pacientes do sexo masculino $0^{1,9,10,11}$. Esse achado pode se dever ao fato de o hospital onde estão localizadas as UTIs do estudo ser referência no tratamento de algumas doenças crônicas progressivas que tem maior prevalência na população feminina, como é o caso do Lúpus Eritematoso Sistêmico (LES).
Com relação à idade, as pesquisadoras observaram a predominância de duas faixas etárias, que foram a de 58 a 77 anos (37,8\%), seguida da de 18 a 37 anos (35,5\%). A predominância da primeira faixa etária condiz com estudos nacionais ${ }^{10-11}$, na medida em que abrange indivíduos idosos que são a maioria, em se tratando de UTIs. Tal fato pode ser evidenciado em um estudo metodológico realizado em UTI geral, de adultos, pertencente a um hospital privado de nível terciário do Município de São Paulo, no qual a média de idade foi de 65,8 anos $^{10}$, e no estudo realizado com 600 pacientes de UTIs gerais de dois hospitais públicos e dois 
privados também do Município de São Paulo, no qual a idade média dos pacientes que compuseram a amostra foi de 60,76 e a mediana de 62 anos $^{11}$. Esse último estudo afirma, ainda, que a população de idosos tem aumentado progressivamente no Brasil, assim como em outros países, e a vivência clínica dos enfermeiros tem demonstrado a elevação do número de idosos em UTI em consonância com o aumento dessa população.

Possíveis explicações, vivenciadas em Teresina-PI, que poderiam justificar a grande quantidade de pacientes na faixa etária de 18 a 37 anos, seriam: número elevado de internação de pós-operatório imediato (cirurgias ortopédicas como artrodese de coluna, por exemplo); as decorrentes de acidentes automobilísticos; a elevação da incidência das doenças crônicas progressivas, das doenças cardiovasculares, como aneurisma e acidente vascular encefálico (AVE), em adultos jovens.

Quanto ao tempo de internação, a média foi de sete dias, sendo que a maioria $(66,7 \%)$ permaneceu internada de dois a oito dias, corroborando um estudo realizado em uma UTI geral de um hospital filantrópico de Teresina, no qual a maior parcela $(84,85 \%)$ dos pacientes permaneceu internada de dois a sete dias ${ }^{12}$, e o estudo desenvolvido em UTI de adultos do Hospital Universitário da Universidade de São Paulo, no qual $64,0 \%$ dos pacientes permaneceram na UTI por um período de um a cinco dias ${ }^{13}$, o que remete a um planejamento para uma demanda alta de pacientes graves e com pouca rotatividade.

As principais procedências foram de outras Unidades de Internação do próprio hospital (44,5\%) e do Centro Cirúrgico (33,3\%), diferindo em certa proporção de alguns estudos nacionais ${ }^{1,14}$ pelo fato de o hospital não disponibilizar de serviço de Pronto-Socorro (sendo estes referenciados de outro Hospital Público) nem de Unidade Semi-Intensiva (USI). No que concerne ao tipo de internaçã̃o, foi predominantemente clínico (62,3\%), seguido do cirúrgico, com $37,7 \%$, sendo $24,4 \%$ das cirurgias eletivas e 13,3\% de urgência, assemelhando-se a estudos nacionais ${ }^{1,14}$.

Quanto ao motivo da internação, 35,5\% foram por pósoperatório imediato, $28,9 \%$ por doenças cardiovasculares e $20,0 \%$ por doenças respiratórias, estando diretamente relacionadas às faixas etárias predominantes no estudo. Supõese que a principal causa foram as cirurgias ortopédicas, relacionadas a causas externas que são mais incidentes na faixa etária de 18 a 37 anos; as doenças cardiovasculares e respiratórias podem ser mais evidenciadas na faixa etária de 58 a 77 anos, já que se tratam, em sua maioria, de doenças crônicas associadas, principalmente, ao envelhecimento populacional que vem ocorrendo no nosso país.

A taxa de mortalidade de 20,0\% também foi compatível com a realidade nacional de outros estudos, que tiveram variação de $7,4 \%$ a $38,0 \%$ 9,10,13,14 e semelhante ao encontrado em um estudo realizado em UTI geral-adulto da rede privada do município de São Paulo, no qual se obteve uma taxa de óbito de $18,2 \%$.

Com relação aos indicadores do NAS pontuados durante o estudo, em $100 \%$ dos pacientes avaliados, e durante todos os dias do estudo, foram realizados os seguintes cuidados, conforme os indicadores do NAS: 1 - Monitorização e controle; 2 -Investigações laboratoriais: bioquímica e microbiológicas; 3 - Medicação, exceto drogas vasoativas; 4 - Procedimentos de higiene; 6-Mobilização e posicionamento; 7-Suporte de cuidados aos familiares e parentes; e 8 - Tarefas administrativas e gerenciais.

Outros itens que foram pontuados com elevada frequência foram: 17 - Medida quantitativa do débito urinário (97,6\%); 5 - Cuidados com todos os drenos, exceto sonda gástrica $(84,8 \%) ; 9$ - Suporte respiratório $(77,4 \%) ; 11$ Tratamento para a melhora da função pulmonar $(77,1 \%)$; e 21 - Alimentação enteral por sonda gástrica ou outras vias gastrintestinais $(70,1 \%)$. No estudo desenvolvido em cinco UTIs de dois hospitais privados, de nível terciário, de grande e médio porte, do município de São Paulo, os itens mais pontuados foram 1c, 2, 3, 4a, 5, 7a, 8a, 9, 11 e $17^{15}$, e, em outro, anteriormente citado, realizado em UTI geral-adulto da rede privada do município de São Paulo, os itens mais frequentemente pontuados (acima de 80\%) foram 1a, 2, 3, $7 \mathrm{a}, 8 \mathrm{a}, 9$ e $17^{1}$.

Observa-se que os itens que tiveram maior frequência de pontuação nos estudos foram justamente os que concernem às práticas rotineiras em unidades de cuidados intensivos, demonstrando que essas intervenções terapêuticas são executadas, pelo menos, em uma frequência satisfatória e constante, e que eles já tomam grande parte do tempo de atuação da enfermagem em um único plantão.

A literatura ${ }^{16}$ descreve algumas intervenções essenciais para as áreas de especialidade de enfermagem, citando as listadas a seguir dentre as intervenções de enfermagem de cuidados críticos e o tempo necessário para a realização delas: Monitoração de sinais vitais (15 min); Administração de medicamentos: intravenosa (15 min); Posicionamento (16 - 30 min); Promoção do Envolvimento Familiar (Mais de 1 hora); Apoio emocional (16 - 30 min); Documentação (15 min); Monitoração hídrica (16 - 30 min); Oxigenoterapia (15 min); Controle de vias aéreas (16 - 30 min); Aspiração de vias aéreas (15 min); e Controle da Ventilação Mecânica: invasiva/não invasiva (mais de 1 hora). A duração de cada intervenção pode variar de 15 minutos a mais de uma hora, sendo outra forma de se calcular o tempo necessário para os cuidados de enfermagem que determinado paciente requer, de acordo com o conjunto de intervenções planejadas pelo profissional de enfermagem no atendimento ao paciente de forma individualizada.

Infere-se, ainda, que se estabeleceu tempo mínimo suficiente para execução dos cuidados intensivistas, o que, na 
realidade vivenciada, ainda está aquém do preconizado pelas evidências internacionais².

Já entre os itens que não foram pontuados durante a aplicação do NAS nesse estudo, teve-se: $4 c$ - Realização de procedimentos de higiene que dure mais de quatro horas em algum plantão e 14-Monitorização do átrio esquerdo. Outros foram pouco pontuados, a saber: 18 - Medida de pressão intracraniana; 15 - Reanimação cardiorrespiratória; 20 Nutrição parenteral total; 16 - Técnica de hemofiltração, técnicas dialíticas; 13 - Reposição intravenosa de grandes perdas de fluidos; 23 - Intervenções espećficas fora da Unidade de Terapia Intensiva; 22 - Intervenções específicas na Unidade de Terapia Intensiva; e 19 - Tratamento de acidose/alcalose metabólica. Em estudos nacionais com a mesma perspectiva, os itens menos pontuados foram 14, 19 e $1 c^{1}$ e 13, 15, 16, 22 e $23^{12}$, além dos itens $14,18,19$ e 20 , que não foram pontuados $^{12}$. Pode-se perceber que os indicadores que foram pouco pontuados ou não foram, referem-se, em sua maioria, a intervenções mais específicas que não se aplicam a todos pacientes que necessitam de cuidados intensivos, justificando a baixa frequência de pontuação desses itens.

Com relação ao item 15 , reanimação cardiorrespiratória (RCR), nas UTIs estudadas, apesar de nove casos terem evoluído a óbito, esse item foi pontuado apenas para três pacientes (1/ 3 dos óbitos).

A parada cardiorrespiratória (PCR) é um evento que ocorre com frequência em UTI, uma vez que essas unidades assistem pacientes gravemente enfermos, com instabilidade hemodinâmica acentuada, e o enfermeiro intensivista é vital nos esforços para reanimar um paciente, pois é ele, frequentemente, quem avalia em primeiro lugar o paciente $\mathrm{e}$ inicia as manobras de RCR, chamando a equipe ${ }^{17}$.

No que concerne à carga de trabalho de enfermagem, segundo o NAS, que foi obtida após as 328 aplicações desse instrumento aos 45 pacientes, os resultados do estudo demonstraram que, no dia em que algum paciente morre, geralmente a pontuação do NAS é mais elevada, pois este requer mais horas de enfermagem relativas ao preparo do corpo, na oferta de suporte emocional à família (subitem 7b), na realização de tarefas administrativas e gerencias (subitem 8c), referentes à declaração de óbito e doação de órgão, por exemplo, entre outras atividades que são peculiares à gravidade do paciente momentos antes e na ocasião do óbito. Dessa forma, o percentual do NAS variou de $39,2 \%$ a $133,7 \%$, sendo a média total de $67,3 \%$, que foi compatível com estudos nacionais que variaram de $61,9 \%$ a $73,7 \%$ 1,8,9,12,14,15.

\section{CONCLUSÃO}

Os pacientes internados nas UTIs estudadas em Teresina-PI demandavam elevada necessidade de cuidados. Considerando o instrumento NAS, aqueles cuidados pertencentes às categorias de "Atividades básicas", "Tarefas administrativas e gerenciais" e "Suporte respiratório" foram os mais frequentemente encontrados. No entanto, existiram limitações no estudo, relacionadas ao número reduzido da amostra, assim como ao método de coleta dependente das anotações de enfermagem, que podem ter implicado uma demanda de cuidados possivelmente menor que a real, em virtude de um provável sub-registro dos cuidados realizados.

Atualmente muitos relatos são ouvidos nas unidades hospitalares sobre o distanciamento dos profissionais enfermeiros da assistência; isso parece ser confirmado ao passo que os itens de gerenciamento e administrativo do NAS foram bastante pontuados, o que alerta a necessidade emergente da presença, nessas unidades, de enfermeiros administrativos e enfermeiros assistencialistas.

As autoras sugerem que os enfermeiros assistencialistas apliquem o NAS na visita diária de enfermagem para evitar um dimensionamento subestimado ou superestimado, para avaliação das condições clínicas dos pacientes, e não apenas a quantidade de internação.

A partir das informações colhidas, por meio da aplicação desse instrumento, é possível verificar se as equipes de enfermagem presentes nas unidades hospitalares estudadas estão condizentes com a demanda de cuidados requerida pelos pacientes e, com isso, aprimorar o dimensionamento destes profissionais. Destarte, esta pesquisa confirma a indicação do NAS como instrumento de gerenciamento de recursos humanos e como auxiliar no planejamento da assistência, a partir de uma adequação numérica da equipe de enfermagem e da quantificação das intervenções prestadas.

Sugere-se ainda que outros estudos sejam realizados utilizando-se o NAS para verificar sua aplicabilidade e a demanda de cuidados de enfermagem em outras realidades brasileiras.

\section{REFERÊNCIAS}

1 Conishi RMY, Gaidzinski RR. Nursing Activities Score-NAS como instrumento para medir carga de trabalho de enfermagem em UTI adulto. Rev Esc Enferm USP. 2007; 41(3): 346-54.

2 Cintra EA, Nishide VM, Nunes WA. Assistência de enfermagem ao paciente gravemente enfermo. $2^{a}$ ed. São Paulo: Atheneu; 2008.

3 Silva RC, Ferreira MA. A tecnologia em saúde: uma perspectiva psicossociológica aplicada ao cuidado de enfermagem. Esc Anna Nery. 2009; 13(1): 169-73.

4 Atkinson LD, Murray ME. Fundamentos de enfermagem: introdução ao processo de enfermagem. Rio de Janeiro: Guanabara Koogan; 2008.

5 Souza SROS, Silva CA, Mello UM, Ferreira CN. Aplicabilidade de indicador de qualidade subjetivo em terapia intensiva. Rev Bras Enferm. 2006 marabr; 59(2): 201-05. 
6 Tranquitelli AM, Padilha KG. Sistemas de classificação de pacientes como instrumentos de gestão em Unidades de Terapia Intensiva. Rev Esc Enferm USP. 2007 mar; 41(1): 141-46.

7 Queijo AF, Padilha KG. Nursing Activities Score- NAS: adaptação transcultural e validação para a língua portuguesa. Rev Esc Enferm USP. 2009; 43( n esp.): 1009-016.

8 Castro MCN, Dell'Acqua MCQ, Corrente JE, Zornoff DCM, Arantes LF. Aplicativo informatizado com o Nursing Activities Score: instrumento para gerenciamento da assistência em unidade de terapia intensiva. Texto \& Contexto Enferm. 2009 set; 18(3): 577-85.

9 Ducci AJ, Zanei SSV, Whitaker IY. Carga de trabalho de enfermagem para quantificar proporção profissional de enfermagem/paciente em UTI cardiológica. Rev Esc Enferm USP. 2008 dez; 42(4): 673-80.

10 Ducci AJ, Padilha KG. Nursing Activities Score: estudo comparativo da aplicação retrospectiva e prospectiva em unidade de terapia intensiva. Acta Paul Enferm. 2008; 21(4): 581-87.

11 Sousa RMC, Padilha KG, Nogueira LS, Miyadahira AMK, Oliveira VCR. Carga de trabalho de enfermagem requerida por adultos, idosos e muito idosos em Unidade de Terapia Intensiva. Rev Esc Enferm USP. 2009 dez; 43( n esp 2): 1284-91.

12 Leite IRL. A demanda de trabalho de enfermagem em Unidade de Terapia Intensiva [monografia]. Piauí: Departamento de Enfermagem, Universidade Federal do Piaú; 2010.

13 Ciampone JT, Gonçalves LA, Maia FOM, Padilha KG. Necessidades de cuidados de enfermagem e intervenções terapêuticas em Unidade de Terapia Intensiva: estudo comparativo entre pacientes idosos e não idosos. Acta Paul Enferm. 2006 jan-mar; 19(1): 28-34.

14 Gonçalves LA, Garcia PC, Toffoleto MC, Telles SCR, Padilha KG. Necessidades de cuidados de enfermagem em terapia intensiva: evolução diária dos pacientes segundo o Nursing Activities Score- NAS. Rev Bras Enferm. 2006 jan-fev; 59(1): 56-60.

15 Gonçalves LA, Padilha KG. Fatores associados à carga de trabalho de enfermagem em Unidade de Terapia Intensiva. Rev Esc Enferm USP. 2007 dez; 41(4):1-11.

16 Bulechek GM, Butcher HK, Dochterman JM. Classificação das Intervenções de Enfermagem- NIC. $5^{\text {a }}$ ed. Rio de Janeiro: Elsevier; 2010.

17 Zanin J, Nascimento ERP, Barra DCC. Parada e reanimação cardiorrespiratória: conhecimentos da equipe de enfermagem em Unidade de Terapia Intensiva. Rev Bras Ter Intensiva. 2006 abr-jun; 18(2): 14347. 